\title{
Fast capillary discharge plasma as a preformed medium for longitudinally pumped collisional x-ray lasers
}

\author{
K. A. Janulewicz, F. Bortolotto, A. Lucianetti, W. Sandner, and P. V. Nickles \\ Max Born Institute, Max-Born-Strasse 2A, D-12489 Berlin, Germany \\ J. J. Rocca \\ Department of Electrical and Computer Engineering, Colorado State University, Fort Collins, Colorado 80523
}

N. Bobrova and P. V. Sasorov

Institute of Theoretical and Experimental Physics, 117259 Moscow, Russia

Received April 24, 2002; revised manuscript received September 4, 2002

\begin{abstract}
Simulations of plasma dynamics in a fast capillary discharge are presented. The temporal dependence of the plasma column's resistance validates the one-dimensional model that was used in the numerical simulations. Numerical analysis of the laser absorption determines the pump parameter range for efficient excitation of longitudinally pumped transient collisional x-ray lasers. () 2003 Optical Society of America
\end{abstract}

OCIS codes: $140.7240,140.5560$.

\section{INTRODUCTION}

A plasma slab with well-defined and controlled parameters that permit guiding of a driving laser beam is a prerequisite for an efficient $\mathrm{x}$-ray laser (XRL). It was demonstrated during the past decade that a double-pulse or a multipulse laser pumping technique efficiently reduces pump energy requirements. A significant improvement in XRL output was achieved when a pumping scheme that combines a long (nanosecond) with a short (picosecond) laser pulse was proposed ${ }^{1}$ and demonstrated. ${ }^{2}$ This pumping method resulted in a high and short-lived (transient) inversion in collisionally pumped XRLs. Owing to the separation of plasma ionization from the heating process it became possible to synchronize the onset of excitation with the optimum ionization state. ${ }^{3}$ Hence control over plasma preparation before the heating phase (plasma preforming) became a crucial component of the transient scheme. Performance relies on preparation of a plasma with a prescribed ionization stage and density. The preplasma temperature is not critical. Plasma preforming for a germanium Ne-like x-ray laser in a slab target geometry was comprehensively investigated numerically in Ref. 4. As this slab target arrangement is plagued by strong refraction on steep density gradients, which results in reduction of the active medium's effective length, density gradient softening, for example by a prepulse $^{5,6}$ or a guiding ${ }^{7}$ technique, was proposed. Much research was recently done on plasma guiding of highand ultrahigh-intensity laser pulses, i.e., on confining an intense laser beam during propagation within an area of limited cross section. ${ }^{8-11}$ However, application of this technique to $\mathrm{x}$-ray lasers was discussed relatively seldom.
Plasma channels for efficient XRL pumping can be created in a gas that is either confined in a limited volume of a free space ${ }^{8}$ or fills a capillary channel. ${ }^{11}$ Such a channel can also be created by material ablation of a capillary wall. The capillary discharge or a long laser pulse delivers the energy needed for ablation. ${ }^{10,12}$ All these methods lead to a concave radial electron density profile with its minimum on the pump beam's axis, which is a precondition of the guiding effect.

In the present paper we concentrate on preforming with a fast capillary discharge, specifically, on a modified version of the process that was developed by Rocca ${ }^{12-15}$ for discharge-pumped collisional XRLs. This kind of fast discharge gives a well-sustained, highly ionized plasma that is particularly suitable as a preformed medium for laser pumped collisional XRLs. Lasing at $60.8 \mathrm{~nm}(\mathrm{Ne}-$ like sulfur) has already been demonstrated ${ }^{16}$ in a hybrid (electrically preformed and optically heated) $\mathrm{x}$-ray laser in a solid sulfur capillary. A gain coefficient of $4.6 \mathrm{~cm}^{-1}$ was measured for a 1-cm-long capillary, but longer capillaries amplified the x-ray signal much less well. Gain coefficients of 1.2 and $1 \mathrm{~cm}^{-1}$ were estimated for the second and third 1-cm-long sections, respectively, of a 3-cm-long capillary with a diameter of $0.5 \mathrm{~mm}$. The total gainlength product was 6.8 , and the total pump energy was $\sim 2.2 \mathrm{~J}(0.3-0.5 \mathrm{~J}$ in the laser pulse). Reduction of the divergence of the emitted radiation with capillary length, to $2.5 \mathrm{mrad}$ for a $3-\mathrm{cm}$ capillary, has confirmed that lasing was observed. A battery with capacity of $0.08 \mu \mathrm{F}$ charged to $4-8 \mathrm{kV}$ gave a discharge peak current of 3-8 $\mathrm{kA}$, depending on capillary dimensions.

This pumping scheme appeared to be technically com- 
plex compared with that of a solely discharge-pumped XRL but was highly efficient. The pumping scheme's efficiency, commonly expressed as a ratio of the gain-length product to the total pump energy, was $3.1 \mathrm{~J}^{-1}$, one of the highest ever measured for collisionally pumped transient $\mathrm{x}$-ray lasers (typically $g l=1-2$ ). Moreover, instead of the preforming laser pulse energy, we took the total electric energy dissipated in the discharge to calculate this parameter. Thus a considerable improvement in total efficiency could be expected.

The experiment confirmed that low energy is necessary for preforming and exciting the active medium of a hybrid XRL. ${ }^{17}$ However, the active medium's length in such a longitudinally pumped scheme could be limited by depletion of the axial pump's radiation as a result of absorption dominated by an inverse bremsstrahlung (IB) mechanism. The plasma has to be heated and ionized as uniformly as possible along the axis by the short laser pulse, as any cold spot in the plasma would interfere with lasing by increasing the absorption of the amplified radiation. This problem is more general and will be encountered in all collisional schemes in which the longitudinal pump arrangement is applied. Another potential problem is walk-off of the propagating pumping and emitted radiation caused by gain-induced differences in the dispersion for the amplified radiation. This problem has to be treated more generally and is beyond the scope of this paper.

The configuration with a density-guided pumping laser pulse within a concave radial distribution of the plasma has two apparent advantages. First, the traveling-wave arrangement is implemented inherently and, second, refraction is eliminated. Recently the plasma dynamics in a discharge-ablated capillary driven by a short electric pulse was analyzed both experimentally and theoretically. ${ }^{14}$ Our results with respect to the plasma dynamics are complementary to those presented there.

\section{RESULTS}

Plasma used as a preformed medium for a hybrid XRL should fulfill, as mentioned above, two basic criteria. First, it has to be ionized to the required stage, and second, it has to possess a density distribution that is suitable for guiding the pump laser pulse. The former factor is not critical because it is possible to improve the average ionization stage during the heating phase by direct field ionization of the lightly ionized ions interacting with an intense main pumping pulse. The overionization that is due to intensity that is higher than the threshold intensity for the appearance of F- (Co-)like ions determines a limit on the pump laser pulse's intensity. These ionization thresholds for S VII and Ti XIII are $6.9 \times 10^{17}$ and $1.1 \times 10^{19} \mathrm{~W} / \mathrm{cm}^{2}$, respectively, and are far above the usually applied pump intensities. An active medium with nearly $100 \%$ of the species ionized to the required stages could be achieved by moderate pump intensities because the thresholds for appearance of Ne-like ions are significantly lower $\left(9.8 \times 10^{15}\right.$ and $2.4 \times 10^{17} \mathrm{~W} / \mathrm{cm}^{2}$ for sulfur and titanium, respectively) than that for the next ionization stage. We modeled the discharge in a sulfur capillary under conditions that correspond to those in ef- fect when lasing was observed. ${ }^{16}$ We used the plasma resistance measurement to validate the theoretical model, as more-sophisticated methods were not available because of the compact design of the discharge unit and the ensuing difficulties in gaining access to the plasma channel.

\section{A. Plasma Dynamics}

The one-dimensional (1D) model described in detail in Ref. 18 was used in the simulations. Three capillary diameters $(0.3,0.5$, and $1 \mathrm{~mm})$ were considered, but length was not specified in the $1 \mathrm{D}$ model. The experimentally registered current waveform in a 20 -mm-long sulfur capillary was critically damped (no current oscillations were observed), which suggested optimal coupling of the electric energy into the discharge. This waveform, with a peak value of $3 \mathrm{kA}$ and a total duration of $250 \mathrm{~ns}$, was approximated by an empirical formula: $I(t)[\mathrm{kA}]=1.1$ $\times(t / 20) \exp \left[-t / 240-(t / 138)^{2}\right]$, where time is expressed in nanoseconds. Both theoretical and experimental shapes of the plasma resistance as well as the discharge current are shown in Fig. 1. The experimental resistance was estimated from the impedance measurement corrected by the self-inductance estimates obtained in a separate experiment. In this experiment the battery was discharged through a silver wire of $0.5-\mathrm{mm}$ diameter and 3 -cm length (the so-called ideal conductor) that filled the capillary channel. As we know the electric characteristics of the silver and the frequency of the signal oscillations, it was possible to determine the resistance of the wire. Constant self-inductance (in a zero-order approximation) during the whole discharge was assumed. Using this information, we could extract the resistance of the discharge plasma from the impedance measurements. It can be seen from Fig. 1 that only the initial and high current phases of the discharge resistance are reproduced with good accuracy by the model. The split between these phases increases with time and begins shortly after the peak of the current discharge. The calculated resistance for the sulfur capillary is $1.35 \Omega$ at the current peak, and the measured value is $1.3 \Omega$. These values agree very well with the result of $1.3 \Omega$ that we obtained by applying the formulas given in Ref. 19 .

We used the same formulas to estimate the discharge circuit's inductance, and the resultant value of $0.1 \mu \mathrm{H}$ deviates from the experimental value of $0.04 \mu \mathrm{H}$. Some possible reasons for that deviation are given in what fol-

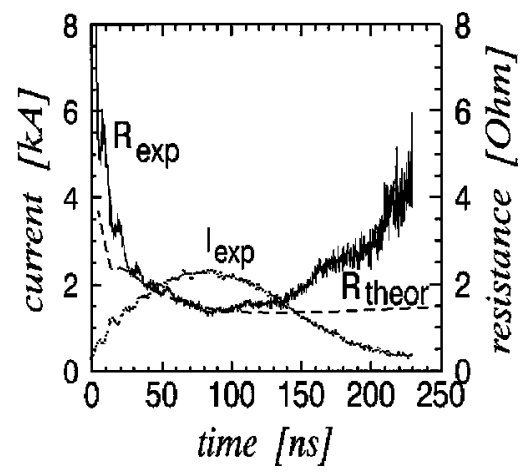

Fig. 1. Time history of the discharge circuit's resistance for a sulfur capillary of $20-\mathrm{mm}$ length. 


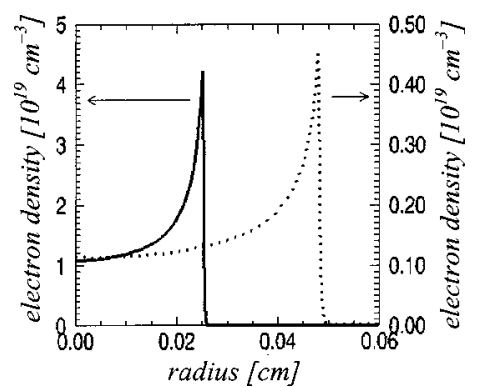

Fig. 2. Radial electron density distributions in the capillaries with diameters of 0.5 and $1.0 \mathrm{~mm}$ at the optimal moments for lasing.

lows. It is well known that this resistance is controlled primarily by the plasma's temperature and only slightly by the plasma's density. The impedance measured is an averaged one and is determined by the hotter part of the plasma because of the parallel connection (analogous to an electric circuit) of various plasma regions. We did not observe any fast change in the resistance during nearly the whole high-current period. The biggest deviation from the theoretical value observed within the time gap of $120 \mathrm{~ns}$, which is interesting from the point of view of guidance, was $0.15 \Omega$. A stronger deviation from the experimental shape that appeared after $150 \mathrm{~ns}$ and increased with time could be caused by the simplified equation of state that was used. This equation of state does not include the influence of ablation on the capillary geometry. More-sophisticated equations of state that include thermodynamic properties of the material make the simulations extremely time consuming. Another plausible explanation for the difference between the theory and experiment is the influence of the plasma outflow effect, which is not included in the 1-D model but could be noticeable at the end of the discharge. Such an explanation could be validated by the measurement of resistance of $\mathrm{Ti}$ capillaries with different lengths but the same discharge current. A shorter capillary $(5 \mathrm{~mm})$ showed, at the end of the discharge, a rate of the impedance increase of more than twice that for the longer capillary $(10 \mathrm{~mm})$. Satisfactory agreement between theory and experiment for sulfur during the first $150 \mathrm{~ns}$ implies that our model used in the simulations is fairly well justified. The discharge current's waveform depends strongly on capillary impedance that is determined, at given applied voltage, by the capillary material and the aspect ratio of the channel. However, any noticeable changes in the discharge resistivities were observed much later than the cutoff moment and were unimportant in the hybrid XRL scheme.

Figure 2 shows theoretical concave electron density profiles with the minimum on the axis that ensures guiding of the pump laser beam. The density on axis increases markedly with decreasing capillary diameter. The density value of $10^{18} \mathrm{~cm}^{-3}$ on axis for a $1-\mathrm{mm}$ diameter capillary could explain the failure of our attempt to observe lasing from such a capillary. This density level is too low to ensure efficient heating of the electrons, which are heated predominantly by the IB absorption mechanism. The electron temperature of $120 \mathrm{eV}$ estimated in the numerical simulation is too low to overcome the energy gap of $192 \mathrm{eV}$ between the ground state and the upper laser levels in the medium. The plasma density in the capillary with a halved diameter is 1 order of magnitude greater, i.e., $1.2 \times 10^{19} \mathrm{~cm}^{-3}$. The electron temperature increases because of the increased absorption to above $200 \mathrm{eV}$.

A qualitatively estimated experimental shape of the density profile is shown in Fig. 3. Having assumed proportionality between the emission in the visible range of the spectrum and the local ion density, we were able to obtain a time-resolved radial density emission profile by imaging the plasma emission at the capillary's output onto the streak camera's slit. A well-defined concave density profile was obtained at a time (29 ns after the current peak) that corresponded to the optimal guiding conditions that were found $35 \mathrm{~ns}$ after the current peak. The value of $35 \mathrm{~ns}$ was estimated from the shots accompanied by lasing. ${ }^{16}$ The plasma emission image shown in Fig. 3 gives only a qualitative picture, as the emission of other deeper channel areas contributed to the background radiation and in this way decreased the dip in the emission profile registered.

The temporal behavior of the electron temperature differs only slightly quantitatively from that presented in Ref. 14. This small difference is understandable, as different capillaries with different discharge parameters were modeled. Additionally, different descriptions of the cooling mechanisms were implemented in the two models. As a cooling model we applied a classic picture described by a generalized form of Braginskii's equations, ${ }^{20}$ and inhibition of thermal conduction with an arbitrarily chosen value $f$ of the inhibition factor was assumed in Ref. 14 . That value could cause the dominance of radiative effects. We assume that the temperature gradients are moderate, and the following validation condition for the classic model, $\lambda_{e} \ll \lambda_{T}$, i.e., that the mean free-electron path is much smaller than the scale length of the temperature gradient, is not violated. Our results also suggest that the plasma pipe in a $0.5-\mathrm{mm}$-diameter capillary is complete after $\sim 50 \mathrm{~ns}$ and that a well-formed channel is already present $20 \mathrm{~ns}$ after onset of the current (Fig. 4). It has to be stressed here that any $1 \mathrm{D}$ model and the following conclusions are invalid for times shorter than $\sim 10 \mathrm{~ns}$. This fact is related to the irregular plasma behavior at the beginning of the capillary discharge. Individual stripes (channels) have been observed experimentally ${ }^{13,14}$ directly after the breakdown, and this phase of the discharge development can be correctly modeled only by a three-dimensional model. The simulations of the plasma dynamics performed for the sulfur capillary also confirm the reduction in axial current. We suspect that less than

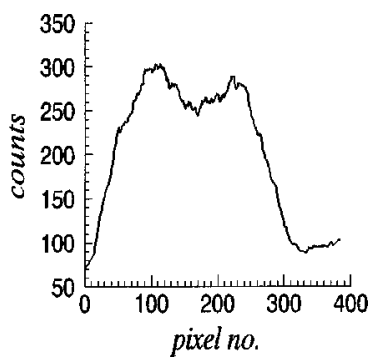

Fig. 3. Measured radial profile of the discharge emission in the visible spectral range $29 \mathrm{~ns}$ after the current's peak. 


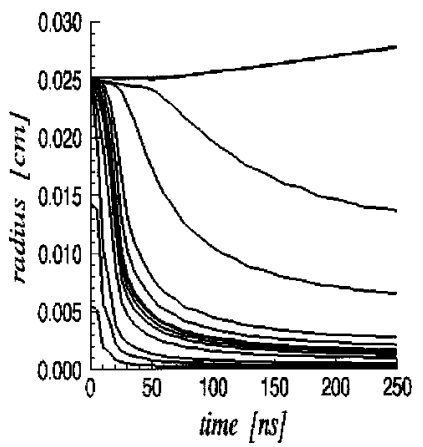

Fig. 4. Movement of the ablated plasma mass elements within the radius-time $(r-t)$ coordinates.

$3 \%$ of the total current flowing in the axial direction is enclosed in a cylinder with a diameter of $50 \mu \mathrm{m}$. This assumption implies that the plasma in the vicinity of the capillary axis is barely magnetized and as a consequence is resistant to magnetohydrodynamic instabilities. Thus hydrodynamics is the dominant mechanism for plasma dynamics in axial proximity.

\section{B. Pump Laser Absorption}

Propagation of the pump radiation and the resultant excitation of the medium in the plasma channel require optimal coupling of the beam energy into the plasma pipe. We have estimated from the numerical results, using the formula $r_{L}=\left(r_{\mathrm{ch}} / \pi r_{e} \Delta n\right)^{1 / 4}$ given in Ref. 9, that the optimum diameter of a Gaussian beam is $\sim 40 \mu \mathrm{m}$. Here $r_{L}$ is the laser spot radius, $r_{e}$ is the classic electron radius, and $r_{\mathrm{ch}}$ together with $\Delta n$ determines the plasma's density profile in the channel $\left[n(r)=n_{0}+\Delta n r^{2} / r_{\mathrm{ch}}{ }^{2}\right]$. It should be noted that the lasing effect was observed with a much broader pumping beam $(d=170 \mu \mathrm{m})$ as a result of technological constraints. These constraints also caused the transmission of the total pump laser beam at the optimal timing to be very low (a few percent). ${ }^{17}$ The effect of heating by IB absorption is estimated with analytical formulas derived by Pert. ${ }^{21}$ The results are valid only for the vicinity of the axis, as no dependence on the laser beam profile is included. One could conclude from the curves shown that pulses with higher intensity are more beneficial for plasma heating (Fig. 5). However, longer pulses (of the order of picoseconds) are preferred because they facilitate energy transfer by collisions. A balance between the two kinds of pulse has to be found, as stronger absorption in the case of longer pulses shortens the length of efficient interaction. As a consequence the increase in intensity has to be achieved rather by the laser beam's being more tightly focused than by pulse shortening. Thus the requirements for efficient coupling of the pump energy to the plasma channel mentioned above are well satisfied, and finally we can infer that the hybrid scheme for Ne-like sulfur that requires picosecond pump laser pulses with energies of 100-150 mJ could work with an increased repetition rate, as such a rate is easily accessible at this low energy level. These energy requirements could be increased on the way toward producing shorter wavelengths, as the elements with higher atomic number $Z$ (titanium, for example) will have to be included and the heating rate (collisional energy transfer) depends on $Z^{*}$ (the average ionization stage). The curves presented in Fig. 5(a) were calculated for a sulfur capillary with the assumption of a $100 \%$ Ne-like sulfur content (average ionization stage, $Z^{*}=6$ ) in the plasma and different pump pulse intensities. The benefit of higher intensities' being applied is uniform heating of the plasma in the axial direction. In fact, the average initial ionization stage is lower than that assumed in the simulations, as the real preformed plasma consists of a mixture of different ionization stages. The axial electron energy distribution in such a case (arbitrarily chosen $50 \% \mathrm{~S} \mathrm{~V}, 30 \% \mathrm{~S}$ VI, and $20 \% \mathrm{~S}$ VII are assumed to be present in the preformed plasma and give an effective $Z^{*}=4.7$ ) and for the constant ionization stage of $Z^{*}=5.5$ is shown in Fig. 5(b). Different final ionization stages and electron energies are obtained, depending on the pump intensity applied. A high intensity, above or close to the intensity for the appearance of S VII, gives a relatively uniform axial distribution of the electron energy, even if the expected maximum ionization stage has not been achieved. In contrast, a relatively high initial ionization stage but with a low laser intensity irradiation gives a highly nonuniform distribution. The influence of the above-thresholdionization (ATI) process on the total electron energy is negligible (1\%) due to very low ionization residual energy. From the results presented here it can be concluded that for the sulfur capillaries an intensity higher than $10^{16} \mathrm{~W} / \mathrm{cm}^{2}$ is required. Then, even an initial ionization stage as low as $Z^{*}=4.7$ could produce nearly $100 \% \mathrm{Ne}$ like sulfur ions over the whole length of the active medium.

Experiments in which subpicosecond laser pulses of high intensity were used to pump collisional XRLs have

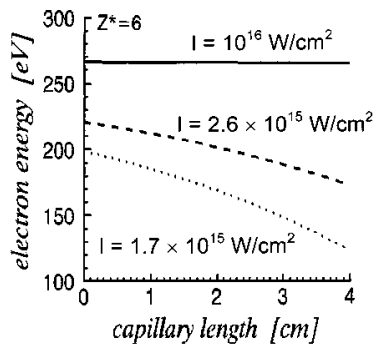

(a)

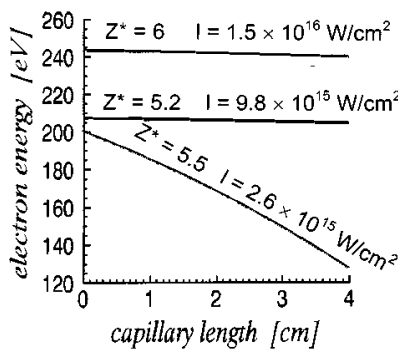

(b)

Fig. 5. (a) Axial distribution of electron energy as a function of the pump intensity. The laser pulse is characterized by its intensity: $Z^{*}=6$ is the initial and final ionization stage; i.e., no ionization has been included. The pulse length is 1 ps. (b) Axial distribution of the electron energy for a mixture of ion species. Initial ionization stage $Z^{*}$ was 4.7 , and the quoted values correspond to the final ionization stages. The curve denoted $Z^{*}=5.5$ corresponds to the constant ionization stage during heating. 


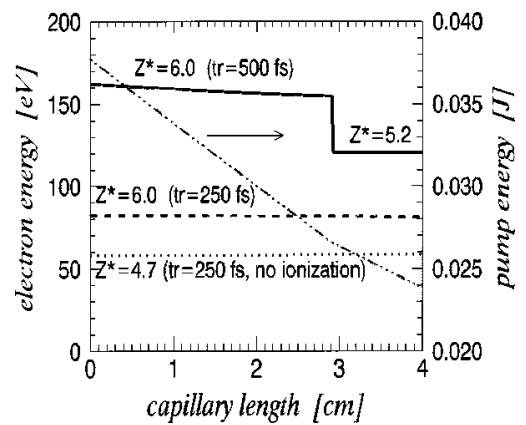

Fig. 6. Axial distribution of electron energy as a function of propagation length. All three pump Gaussian pulses are characterized by the same FWHM $=250 \mathrm{fs}$ and different rise times: $250 \mathrm{fs}$ (dotted curve), $350 \mathrm{fs}$ (dashed curve), and $500 \mathrm{fs}$ (solid curve). The dotted-dashed curve shows axial energy depletion of the pump laser pulse.

recently been reported. We believe that this is not beneficial for efficiency of the scheme, even if some experiments were successful in demonstrating lasing with such pulses. The main problem in a reliable comparison is in achieving unambiguous characteristics of the pump laser pulse. Use of the FWHM as a characteristic parameter of laser-pulse duration, as commonly occurs, is not sufficient to describe the interaction process correctly, as the FWHM does not characterize both the lengths of the two edges and the total pulse length. This problem was discussed in Ref. 22 from the point of view of the ATI. Here we turn our attention to the importance of morecomprehensive pump pulse characterization in the IB absorption process. Gaussian laser pulses with the same peak intensity, $10^{16} \mathrm{~W} / \mathrm{cm}^{2}$, and FWHM, $250 \mathrm{fs}$, but different lengths of the leading edges were used in the numerical simulation. The total pulse length was equal to twice the rise time. The final electron temperature of the heated medium and the final ionization stage were the measures of interaction efficiency. The result is shown in Fig. 6. There is a dramatic difference in the behavior of the plasma irradiated with these laser pulses. The pulses with the shortest total length give the lowest electron temperature and ionization stage. Plasma heating increases with pulse length, but the low pulse energy that results from the subpicosecond pulse length is quickly reduced by the strong IB absorption, and even the longest pulse tested was not able to ionize and heat the medium uniformly.

\section{SUMMARY}

In conclusion, we have analyzed some aspects of using capillary discharge as the source of a preformed plasma for collisionally pumped x-ray lasers in a longitudinal arrangement. A picosecond laser pulse of moderate or low energy irradiated the discharge plasma, and the beam transmitted through the capillary channel during the discharge was monitored. The plasma channel transmission decreased quickly during 80-90 ns after the current peak. Even if it is technologically difficult, the system that we have discussed showed a noticeable increase in pumping efficiency. A significant part of the beam attenuation is caused by the strong IB absorption at mod- erate $\left(\sim 10^{15} \mathrm{~W} / \mathrm{cm}^{2}\right)$ intensities. An intensity increase above the threshold (appearance) intensity of Ne-like ions could give relatively uniform heating in the axial direction. The intensity increase should rather be achieved by tighter pump beam focusing. As a consequence, the pump energies could be reduced to a level that would permit an increased repetition rate (a few hundred millijoules) to be used. The temporal history of the discharge resistance served to validate the model used in the numerical simulations. The behavior of the discharge was in reasonable agreement with the numerical model, at least before and during lasing. The cross section of the visible emission streak demonstrated a concave radial density profile with its minimum on the capillary axis.

\section{ACKNOWLEDGMENTS}

This research was supported by the Deutsche Forschungsgemeinschaft as part of the project "Interaction of the Intense Laser Radiation with Matter" and by the European X-Ray Lasers Network. We are grateful to A. P. Juna for his work on the manuscript.

K. A. Janulewicz's e-mail address is kaj@mbi-berlin.de.

\section{REFERENCES}

1. Yu. V. Afanas'ev and V. N. Shlyaptsev, "Formation of a population inversion of transitions in steady-state and transient plasmas," Quantum Electron. 19, 1606-1612 (1989)

2. P. V. Nickles, V. N. Shlyaptsev, M. P. Kalachnikov, M. Schnuerer, I. Will, and W. Sandner, "Short-pulse x-ray laser at $32.6 \mathrm{~nm}$ based on transient gain in Ne-like titanium," Phys. Rev. Lett. 76, 2748-2751 (1997).

3. S. B. Healy, K. A. Janulewicz, J. A. Plowes, and G. J. Pert, "Transient high gains at $196 \AA$ produced by picosecond pulse heating of a preformed germanium plasma," Opt. Commun. 132, 442-448 (1996).

4. K. A. Janulewicz, S. B. Healy, M. P. Kalachnikov, P. J. Warwick, P. V. Nickles, W. Sandner, and G. J. Pert, "Influence of pump pulse parameters on the collisionally pumped germanium x-ray laser in the transient gain regime," Opt. Commun. 168, 183-193 (1999).

5. J. Nilsen, B. J. MacGowan, L. B. Da Silva, and J. C. Moreno, "Prepulse technique for producing low-Z Ne-like x-ray lasers," Phys. Rev. A 48, 4682-4685 (1993).

6. S. B. Healy, J. A. Plowes, G. Cairns, C. L. S. Lewis, and G. J. Pert, "A computational investigation of the neon-like germanium collisionally pumped laser considering the effect of prepulses," IEEE J. Sel. Top. Quantum Electron. 1, 949957 (1995).

7. R. Li, T. Ozaki, T. Kanai, and H. Kuroda, "Proposal of the longitudinally pumped saturated Ni-like Mo x-ray laser at 18.9 nm," Phys. Rev. E 57, 7093-7102 (1998).

8. H. M. Milchberg, C. G. Durfee III, and J. Lynch, "Application of plasma channels to soft x-ray lasers," J. Opt. Soc. Am. B 12, 731-737 (1995).

9. Y. Ehrlich, C. Cohen, D. Kaganovich, A. Zigler, R. F. Hubbard, P. Sprangle, and E. Esarey, "Guiding and damping of high-intensity laser pulses in long plasma channels," J. Opt. Soc. Am. B 15, 2416-2423 (1998).

10. Y. Ehrlich, C. Cohen, A. Zigler, J. Krall, P. Sprangle, and E. Esarey, "Guiding of high-intensity laser pulses in straight and curved plasma channel experiments," Phys. Rev. Lett. 77, 4186-4189 (1996).

11. S. M. Hooker, D. J. Spence, and R. A. Smith, "Guiding of high-intensity picosecond laser pulses in a dischargeablated capillary waveguide," J. Opt. Soc. Am. B 17, 90-98 (2000). 
12. D. V. Korobkin, C. Nam, and S. Suckewer, "Demonstration of soft x-ray lasing to ground-state in Li III," Phys. Rev. Lett. 77, 5206-5209 (1996).

13. M. Marconi, C. H. Moreno, J. J. Rocca, V. N. Shlyaptsev, and A. L. Osterheld, "Dynamics of a microcapillary discharge plasma using a soft x-ray laser backlighter," Phys. Rev. E 62, 7209-7218 (2000).

14. J. J. Rocca, V. N. Shlyaptsev, F. G. Tomasel, O. D. Cortazar, D. Hartshorn, and J. L. A. Chilla, "Demonstration of a discharge pumped table-top soft x-ray laser," Phys. Rev. Lett. 73, 2192-2195 (1994).

15. J. J. Rocca, "Table-top soft x-ray lasers," Rev. Sci. Instrum. 70, 3799-3827 (1999)

16. K. A. Janulewicz, J. J. Rocca, F. Bortolotto, V. N. Shlyaptsev, M. P. Kalachnikov, W. Sandner, and P. V. Nickles, "Demonstration of a hybrid collisionally pumped soft x-ray laser," Phys. Rev. A 63, 033803 (2001).

17. K. A. Janulewicz, F. Bortolotto, P. J. Warwick, M. P. Kalachnikov, V. N. Shlyaptsev, W. Sandner, J. J. Rocca, and P. V. Nickles, "Studies of a sulfur capillary discharge irradiated by a ps-laser pulse: a new way towards collisionally pumped table-top x-ray lasers," in Soft X-Ray Lasers and Applications III, J. J. Rocca and L. Da Silva, eds., Proc. SPIE 3776, 37-44 (1999).

18. N. A. Bobrova, S. V. Bulanov, D. Farina, R. Pozzoli, T. L. Razinkova, J. I. Sakai, P. V. Sasorov, and I. V. Sokolov, "MHD simulations of plasma dynamics in pinch discharges in capillary plasmas," Laser Part. Beams 18, 623-638 (2000).

19. R. A. McCorkle, "The high-power sliding-spark capillary discharge in vacuum: variations and applications," Appl Phys. A 26, 261-270 (1981).

20. G. J. Pert, "Inverse bremsstrahlung in strong radiation fields at low temperatures," Phys. Rev. E 51, 4778-4789 (1995).

21. N. A. Bobrova and P. V. Sasorov, "MHD equations for a fully ionized plasma of complex composition," Sov. J. Plasma Phys. 19, 409-421 (1993).

22. K. A. Janulewicz, M. J. Grout, and G. J. Pert, "Electron residual energy of optical-field-ionized plasmas driven by subpicosecond laser pulses," J. Phys. B 29, 901-914 (1996). 